MATHEMATICS OF COMPUTATION

Volume 71, Number 239, Pages 1271-1280

S 0025-5718(01)01403-X

Article electronically published on December 21, 2001

\title{
A COMPUTATIONAL APPROACH TO HILBERT MODULAR GROUP FIXED POINTS
}

\author{
JESSE IRA DEUTSCH
}

\begin{abstract}
Some useful information is known about the fundamental domain for certain Hilbert modular groups. The six nonequivalent points with nontrivial isotropy in the fundamental domains under the action of the modular group for $\mathbf{Q}(\sqrt{5}), \mathbf{Q}(\sqrt{2})$, and $\mathbf{Q}(\sqrt{3})$ have been determined previously by Gundlach. In finding these points, use was made of the exact size of the isotropy groups. Here we show that the fixed points and the isotropy groups can be found without such knowledge by use of a computer scan. We consider the cases $\mathbf{Q}(\sqrt{5})$ and $\mathbf{Q}(\sqrt{2})$. A computer algebra system and a $\mathrm{C}$ compiler were essential in perfoming the computations.
\end{abstract}

\section{INTRODUCTION}

To give perspective on the fixed points of two-dimensional Hilbert modular groups, a quick review of the one-dimensional case is helpful. In the latter case, $\mathbf{H}$ is the upper half of the complex plane and the group of two by two matrices of determinant one with integer entries acts on $\mathbf{H}$ by the corresponding linear fractional transformation. This group is denoted by $\Gamma$. We define a closed subset $F$ of $\mathbf{H}$ to be a fundamental domain for $\Gamma$ only if the following two conditions hold. For every point in $\mathbf{H}$ there exists a point in $F$ that is mapped to it under some transformation in $\Gamma$. Additionally, if there are two points of $F$ that are related by a nontrivial mapping from $\Gamma$, these points must lie on the boundary of $F$. The classic result of the one-dimensional case is that the region $F=\{z \in \mathbf{H}|| z|\geq 1,| \Re z \mid \leq 1 / 2\}$ is a fundamental domain for $\Gamma$. It is also known that points in $F$ that are fixed by nontrivial elements of $\Gamma$ are $i, \rho$ and $-\rho^{-1}$ where $\rho=-1 / 2+i \sqrt{3} / 2$. Note that $\rho$ and $-\rho^{-1}$ are equivalent under the transformation $z \mapsto z+1$, which is in $\Gamma$. Thus there are only two inequivalent points of $F$ that are fixed by nontrivial mappings of $\Gamma$. We will see that these points lift to fixed points in the two-dimensional case.

For the two-dimensional analogue of the modular group we consider the product of upper half planes in $\mathbb{C}^{2}$ (see Gotzky [3, pp. 413-414], Gundlach [4, p. 109]):

$$
\mathbf{H}^{2}=\left\{\left(z, z^{\prime}\right) \mid \Im z>0, \Im z^{\prime}>0\right\}
$$

and the ring of algebraic integers $O$ of a real quadratic number field $D$. We let $\varepsilon$ be the fundamental unit of the ring of algebraic integers. The group of two by two

Received by the editor January 6, 2000 and, in revised form, September 7, 2000.

2000 Mathematics Subject Classification. Primary 11-04, 11Y35; Secondary 32-04.

Key words and phrases. Modular group, fundamental domain, quadratic field.

(C)2001 American Mathematical Society 
matrices of interest, called the Hilbert modular group $\Gamma=\Gamma(O)$, is

$$
\Gamma(O)=\left\{\left(\begin{array}{ll}
\alpha & \beta \\
\gamma & \delta
\end{array}\right) \mid \alpha, \beta, \gamma, \delta \in O, \alpha \delta-\beta \gamma=1\right\} .
$$

The action of this group on $\mathbf{H}^{2}$ is

$$
\left(\begin{array}{ll}
\alpha & \beta \\
\gamma & \delta
\end{array}\right)\left(z, z^{\prime}\right) \mapsto\left(\frac{\alpha z+\beta}{\gamma z+\delta}, \frac{\alpha^{\prime} z^{\prime}+\beta^{\prime}}{\gamma^{\prime} z^{\prime}+\delta^{\prime}}\right),
$$

where $\alpha^{\prime}, \beta^{\prime}, \gamma^{\prime}, \delta^{\prime}$ are conjugates with respect to $O$. The above transformations preserve $\mathbf{H}^{2}$ (see Gotzky [3, p. 416]).

A fundamental domain for the action of $\Gamma$ on $\mathbf{H}^{2}$ is defined in exactly the same way as in the one-dimensional case. Indeed the same definition works for any group whose elements map $\mathbf{H}^{2}$ onto itself. We follow Gotzky's work and denote certain special matrices with the notation

$$
S=\left(\begin{array}{ll}
1 & 1 \\
0 & 1
\end{array}\right), \quad S_{\omega}=\left(\begin{array}{cc}
1 & \omega \\
0 & 1
\end{array}\right), \quad T=\left(\begin{array}{cc}
0 & -1 \\
1 & 0
\end{array}\right), \quad U=\left(\begin{array}{cc}
\varepsilon & 0 \\
0 & \varepsilon^{-1}
\end{array}\right)
$$

where 1 and $\omega$ generate the set of algebraic integers as a $\mathbb{Z}$-module. We note that if the ring of algebraic integers $O$ is norm Euclidean then the modular group $\Gamma(O)$ is generated by $S, S_{\omega}, T$ and $U$. This is essentially proven in Deutsch [2, chapter 4] and is crucial to the construction of the fundamental domain $\mathfrak{S}$ described below.

For a typical element $\left(z, z^{\prime}\right) \in \mathbf{H}^{2}$ let $z=r+i s$ and $z^{\prime}=r^{\prime}+i s^{\prime}$. We define the regions

$$
\mathfrak{U}=\left\{\left(z, z^{\prime}\right) \mid{\varepsilon^{\prime}}^{2} \leq s^{\prime} / s<\varepsilon^{2}\right\}, \quad \mathfrak{T}=\left\{\left(z, z^{\prime}\right)|| z z^{\prime} \mid \geq 1\right\} .
$$

Clearly $\mathfrak{U}$ is a fundamental domain for the group of transformations generated by $U$, and $\mathfrak{T}$ is a fundamental domain for the group of transformations generated by $T$.

Now let $\mathfrak{F}_{s, s^{\prime}}$ be the plane in $\mathbf{H}^{2}$ with $\Im z=s$ and $\Im z^{\prime}=s^{\prime}$. From equivalent points under the transformation group generated by $S$ and $S_{\omega}$ choose those points that minimize $\left|z z^{\prime}\right|^{2}$. See Gotzky [3, p. 417] for more details. Call the set of such points $\mathfrak{S}_{s, s^{\prime}}$. Let

$$
\mathfrak{S}=\bigcup_{s, s^{\prime}>0} \mathfrak{S}_{s, s^{\prime}}
$$

Then $\mathfrak{S}$ is a fundamental domain for the group generated by $S$ and $S_{\omega}$.

Let $F=F(O)=\mathfrak{U} \cap \mathfrak{T} \cap \mathfrak{S}$. Then Gotzky has shown that each point in $\mathbf{H}^{2}$ corresponds to at least one point in $F$ under $\Gamma(O)$-equivalence. See Gotzky [3. p. 418] for details.

Suppose that $\left(z, z^{\prime}\right)$ in $F$ is fixed by an element of $\Gamma$. Then we have the equations

$$
\frac{\alpha z+\beta}{\gamma z+\delta}=z, \quad \frac{\alpha^{\prime} z^{\prime}+\beta^{\prime}}{\gamma^{\prime} z^{\prime}+\delta^{\prime}}=z^{\prime}
$$

Thus

$$
0=\gamma z^{2}+(\delta-\alpha) z-\beta, \quad 0=\gamma^{\prime} z^{\prime 2}+\left(\delta^{\prime}-\alpha^{\prime}\right) z-\beta^{\prime} .
$$

If $\gamma=0$ then $z$ and $z^{\prime}$ are real, which is a contradiction. We may substitute $r+i s$ for $z$ and $r^{\prime}+i s^{\prime}$ for $z^{\prime}$. Taking imaginary parts, we find that

$$
r=\frac{\alpha-\delta}{2 \gamma}, \quad r^{\prime}=\frac{\alpha^{\prime}-\delta^{\prime}}{2 \gamma^{\prime}}
$$


A COMPUTATIONAL APPROACH TO HILBERT MODULAR GROUP FIXED POINTS 1273

Also, by further consideration of imaginary parts

$$
s=\frac{s}{(\gamma r+\delta)^{2}+(\gamma s)^{2}}, \quad s^{\prime}=\frac{s^{\prime}}{\left(\gamma^{\prime} r^{\prime}+\delta^{\prime}\right)^{2}+\left(\gamma^{\prime} s^{\prime}\right)^{2}}
$$

and working with $\left(\Im(-1 / z), \Im\left(-1 / z^{\prime}\right)\right)$, we get

$$
\frac{s}{|z|^{2}}=\frac{s}{(\alpha r+\beta)^{2}+(\alpha s)^{2}}, \quad \frac{s^{\prime}}{\left|z^{\prime}\right|^{2}}=\frac{s^{\prime}}{\left(\alpha^{\prime} r^{\prime}+\beta^{\prime}\right)^{2}+\left(\alpha^{\prime} s^{\prime}\right)^{2}} \text {. }
$$

\section{FIXED POINTS FOR $\mathbf{Q}(\sqrt{5})$}

Let us fix some notation. $O(\sqrt{5})$ is the ring of integers of $\mathbf{Q}(\sqrt{5})$ and $O(\sqrt{2})$ is the ring of integers of $\mathbf{Q}(\sqrt{2})$. In the first case the fundamental unit $\varepsilon$ equals $(1+\sqrt{5}) / 2$, and in the second case $\varepsilon$ is $1+\sqrt{2}$. A typical element $\eta$ of the group $\Gamma(O)$ has entries

$$
\eta=\left(\begin{array}{ll}
\alpha & \beta \\
\gamma & \delta
\end{array}\right)
$$

Again we let $\left(z, z^{\prime}\right)$ be a typical point in $\mathbf{H}^{\mathbf{2}}$ with $z=r+i s$ and $z^{\prime}=r^{\prime}+i s^{\prime}$. We have the key result of Gotzky

Theorem 1 (Gotzky). For $\left(z, z^{\prime}\right) \in \mathfrak{S} \cap \mathfrak{T}$ the product $s \cdot s^{\prime} \geq(\sqrt{312}-9) / 16>$ .54 .

This leads to the results

Theorem 2 (Gotzky). $F(O(\sqrt{5}))$ is a fundamental domain of the modular group for $\mathbf{Q}(\sqrt{5})$. The imaginary parts of a point in this region satisfy $s>.45$ and $s^{\prime}>.45$.

Corollary 1 (Gotzky). An element of $\Gamma(O(\sqrt{5}))$ that maps a point of the fundamental domain $F(O(\sqrt{5}))$ into another satisfies $|N(\gamma)| \leq 1$. If $\gamma \neq 0$, then $\left|z z^{\prime}\right|=1$.

To find the fixed points of $\Gamma(O(\sqrt{5}))$ in the fundamental domain, the basic idea is to place bounds on $\alpha, \beta, \gamma$ and $\delta$ and then do a computer search of all possible eligibile two by two matrices. In order to proceed, we need bounds on $r$ and $r^{\prime}$, the real parts of the coordinates of a point $\left(z, z^{\prime}\right)$ in $F=F(O(\sqrt{5}))$.

Lemma 1. In the fundamental domain $F$ for $\mathbf{Q}(\sqrt{5}),|r|$ and $\left|r^{\prime}\right|$ are each less than 1.853 .

Proof. We use the inequality (see Gotzky [3, p. 420])

$$
\left(r^{2}+s^{2}\right)\left({r^{\prime}}^{2}+s^{2}\right) \leq(5 / 16)^{2}+(9 / 8) s s^{\prime}+\left(s s^{\prime}\right)^{2},
$$

from which it follows that

$$
\begin{aligned}
r^{2}+s^{2} & \leq \frac{(5 / 16)^{2}+(9 / 8) s s^{\prime}+\left(s s^{\prime}\right)^{2}}{r^{\prime 2}+s^{\prime 2}} \\
& \leq \frac{(5 / 16)^{2}+(9 / 8) s s^{\prime}+\left(s s^{\prime}\right)^{2}}{s^{\prime 2}} \\
& \leq(5 / 16)^{2} \frac{1}{s^{\prime 2}}+\frac{9}{8} \frac{s}{s^{\prime}}+s^{2}
\end{aligned}
$$


Hence

$$
r^{2} \leq(5 / 16)^{2} \frac{1}{s^{\prime 2}}+\frac{9}{8} \varepsilon^{2} \leq 3.43357 \ldots,
$$

which gives the bound for $r$. The same argument holds for $r^{\prime}$.

The bounds on the entries of a matrix in $\Gamma(O(\sqrt{5}))$ that fixes a point in the fundamental domain $F$ follow.

Lemma 2. Let $\eta$ be an element of the modular group $\Gamma(O(\sqrt{5}))$ that fixes a point in the fundamental domain $F$. Then

$$
|\alpha|,\left|\alpha^{\prime}\right|<1.86, \quad|\beta|,\left|\beta^{\prime}\right|<5.69, \quad|\gamma|,\left|\gamma^{\prime}\right|<2.23, \quad|\delta|,\left|\delta^{\prime}\right|<5.15 .
$$

Proof. From equation (1.2) we see that

$$
(\gamma r+\delta)^{2}+(\gamma s)^{2}=1
$$

which forces $\gamma^{2} \leq 1 / s^{2}$, which gives $|\gamma|<2.23$ from the bounds on $s$ for a point in the fundamental domain $F$. Similarly, from $(\gamma r+\delta)^{2} \leq 1$ we have

$$
|\delta|-|\gamma r| \leq|\gamma r+\delta| \leq 1
$$

so the bound on $\delta$ can be computed. Since $\left|z z^{\prime}\right|=1$ for any fixed point in $\Gamma$,

$$
|z|^{2} \leq \frac{1}{{r^{\prime}}^{2}+{s^{\prime}}^{2}} \leq \frac{1}{{s^{\prime}}^{2}} .
$$

By (1.3)

$$
\begin{aligned}
& (\alpha r+\beta)^{2}+(\alpha s)^{2}=|z|^{2} \leq \frac{1}{s^{\prime 2}} \\
& \Longrightarrow \alpha^{2} \leq \frac{1}{s^{2} s^{\prime 2}} \quad \Longrightarrow|\alpha|<1.86 .
\end{aligned}
$$

The bound on $|\beta|$ is obtained analogously to the bound on $|\delta|$ :

$$
|\beta|-|\alpha r| \leq|\alpha r+\beta| \leq|z| \leq 2.23 \text {. }
$$

By symmetry, the same bounds hold for $\alpha^{\prime}, \beta^{\prime}, \gamma^{\prime}$ and $\delta^{\prime}$.

Running a computer scan, we obtain the following results.

Theorem 3. There are 94 matrices in $\Gamma(O(\sqrt{5}))$ that correspond to fixed points in the fundamental domain $F$ for $\mathbf{Q}(\sqrt{5})$. The fixed points are at a distance of $\sqrt{2}$ and $\sqrt{3}$ from the origin. The product of the imaginary parts is either $\sqrt{5} / 4,3 / 4$ or 1.

Working with a computer algebra system in addition to further computer scans isolated the inequivalent fixed points of this collection of matrices.

Theorem 4. There are a total of six $\Gamma(O(\sqrt{5}))$ inequivalent fixed points in the fundamental domain $F$ of $\mathbf{Q}(\sqrt{5})$. Two points have cyclic isotropy subgroups of order 4, two points have cyclic isotropy subgroups of order 6, and the final two points have cyclic isotropy subgroups of order 10.

Of the 94 fixed point matrices, all are members of cyclic subgroups of orders 4,6 or 10. There are three subgroups of order 4 , six of order 6 , and eight of order 10 . Of the three subgroups of order 4 , two of the subgroups correspond to fixed points that are $\Gamma(O(\sqrt{5})$ )-equivalent to each other, while the third is not equivalent to either. The six subgroups of order 6 break up into two sets of subgroups which have fixed 
TABLE I. Fixed points in the Fundamental Domain for $\mathbf{Q}(\sqrt{5})$

\begin{tabular}{lll}
\hline Point & Order & Matrix \\
\hline$(i, i)$ & 4 & $\left(\begin{array}{cc}0 & -1 \\
1 & 0\end{array}\right)$ \\
$(i(\sqrt{5}-1) / 2, i(\sqrt{5}+1) / 2)$, & 4 & $\left(\begin{array}{cc}0 & \frac{\sqrt{5}-1}{2} \\
-\frac{\sqrt{5}+1}{2} & 0\end{array}\right)$ \\
$(-1 / 2+i \sqrt{3} / 2,-1 / 2+i \sqrt{3} / 2)$ & 6 & $\left(\begin{array}{cc}0 & -1 \\
1 & 1\end{array}\right)$ \\
$\left(\frac{\sqrt{5}-1}{4}+i \sqrt{3} \frac{\sqrt{5}-1}{4},-\frac{\sqrt{5}+1}{4}+i \sqrt{3} \frac{\sqrt{5}+1}{4}\right)$ & 6 & $\left(\begin{array}{cc}0 & \frac{\sqrt{5}-1}{2} \\
-\frac{\sqrt{5}+1}{2} & 1\end{array}\right)$ \\
$\left(\frac{\sqrt{5}-3}{4}+i \sqrt{2} \frac{\sqrt{5-\sqrt{5}}}{4},-\frac{\sqrt{5}+3}{4}+i \sqrt{2} \frac{\sqrt{5+\sqrt{5}}}{4}\right)$ & 10 & $\left(\begin{array}{cc}\frac{\sqrt{5}-1}{2} & \frac{\sqrt{5}-3}{2} \\
1 & 1\end{array}\right)$ \\
$\left(\frac{\sqrt{5}+3}{4}+i \sqrt{2} \frac{\sqrt{5+\sqrt{5}}}{4}, \frac{3-\sqrt{5}}{4}+i \sqrt{2} \frac{\sqrt{5-\sqrt{5}}}{4}\right)$ & 10 & $\left(\begin{array}{cc}-\frac{\sqrt{5}+1}{2} & \frac{\sqrt{5}+3}{2} \\
-1 & 1\end{array}\right)$ \\
\hline
\end{tabular}

points that are $\Gamma(O(\sqrt{5}))$-equivalent. One set has two subgroups in it, the other has four. The eight subgroups of order 10 break up into two sets of subgroups which have fixed points that are $\Gamma(O(\sqrt{5}))$-equivalent. Each set has four subgroups in it.

For the scan, we write $\alpha=a_{1}+a_{2} \varepsilon$, where $a_{1}$ and $a_{2}$ are rational integers and $\varepsilon$ is the fundamental unit. The inequalities (2.1) for $|\alpha|$ and $\left|\alpha^{\prime}\right|$ give bounds on the sizes of $\left|a_{1}\right|$ and $\left|a_{2}\right|$. Performing a similar representation for the other entries of our typical matrix $\eta$ of $\Gamma(O(\sqrt{5}))$ leads us to 8 "for" loops nested inside each other. Every possible inequality was used to throw out unsuitable candidates, which yielded the 94 remaining matrices.

Table I lists a choice of inequivalent fixed points with corresponding matrices. Another program and a computer algebra system were used to show that the fixed points produced by these matrices were actually in the fundamental domain, i.e., that they satisfied $\left|z z^{\prime}\right| \geq 1$, the condition on the ratio of imaginary parts, and especially the minimum norm condition on all appropriate translates in $\mathfrak{F}_{s, s^{\prime}}$.

\section{Fixed POINTS FOR $\mathbf{Q}(\sqrt{2})$}

Many of the results for $\mathbf{Q}(\sqrt{5})$ have analogues for $\mathbf{Q}(\sqrt{2})$. We now consider the case of $\Gamma(O(\sqrt{2}))$ and fix a fundamental domain, $F$, contained in $F(O(\sqrt{2}))$. Such an $F$ exists due to the results of Gotzky mentioned previously.

Theorem 5. For $\left(z, z^{\prime}\right) \in \mathfrak{S} \cap \mathfrak{T}$ the product $s \cdot s^{\prime} \geq(\sqrt{21}-3) / 4>.395$. 
Proof. This was demonstrated previously (see Gundlach [5, p. 375]) with a slightly different definition of fundamental domain. Alternatively, with some modifications, the proof in Gotzky goes through.

Corollary 2. In $F(O(\sqrt{2}))$ we have $s, s^{\prime}>.26$.

Lemma 3. In $F(O(\sqrt{2})),|r|$ and $\left|r^{\prime}\right|$ are each less than 3.53 .

Proof. The demonstration follows the same pattern as the $\mathbf{Q}(\sqrt{5})$ case.

Unfortunately, some key results of the $\mathbf{Q}(\sqrt{5})$ case do not come through at this point. In particular, there are matrices in $\Gamma(O(\sqrt{2}))$ which map a point in $F(O(\sqrt{2}))$ to itself while $|N(\gamma)|=2$. To find all the matrices of $\Gamma(O(\sqrt{2}))$ that fix a point in $F(O(\sqrt{2}))$, we proceed as follows.

Lemma 4. Let $\eta$ be an element of $\Gamma(O(\sqrt{2}))$ that fixes a point in $F(O(\sqrt{2}))$. Then $|N(\gamma)| \leq 2$.

Proof. By equation (1.2) we have

$$
(\gamma r+\delta)^{2}+(\gamma s)^{2}=1=\left(\gamma^{\prime} r^{\prime}+\delta^{\prime}\right)^{2}+\left(\gamma^{\prime} s^{\prime}\right)^{2},
$$

whence

$$
\begin{aligned}
& 1 \geq \gamma^{2} s^{2}, \quad 1 \geq \gamma^{\prime 2} s^{\prime 2} \\
& \Rightarrow \quad 1 \geq\left(\gamma \gamma^{\prime}\right)^{2}\left(s s^{\prime}\right)^{2} \\
& \Rightarrow \quad N(\gamma)^{2} \leq \frac{1}{\left(s s^{\prime}\right)^{2}} \leq \frac{1}{.395^{2}} \\
& \Rightarrow \quad|N(\gamma)|<3
\end{aligned}
$$

We may again ignore the case of $|N(\gamma)|=0$, as it results in a fixed point with zero imaginary part. The case when the norm is two is dealt with by the following lemma.

Lemma 5. Let $\eta$ be an element of the modular group $\Gamma(O(\sqrt{2}))$ that fixes a point in $F(O(\sqrt{2}))$. If $|N(\gamma)|=2$, then

$$
\alpha=-\delta, \quad \beta=-\frac{\delta^{2}+1}{\gamma} .
$$

Proof. Using (1.1), we find that

$$
\gamma r+\delta=\gamma \frac{\alpha-\delta}{2 \gamma}+\delta=\frac{\alpha+\delta}{2} .
$$

If $\alpha+\delta=0$, then we immediately obtain $\alpha=-\delta$, and the formula for $\beta$ drops out from the equation $\alpha \delta-\beta \gamma=1$.

Suppose $\alpha+\delta \neq 0$. Since $N(\gamma)=2$, it is clear that $\gamma \equiv 0(\bmod \sqrt{2})$. As the order of the factor ring $O(\sqrt{2}) /(\sqrt{2})$ is two, we find that

$$
\begin{aligned}
& \alpha \delta-\beta \gamma=1 \\
\Rightarrow & \alpha \delta \equiv 1(\bmod \sqrt{2}) \\
\Rightarrow & \alpha \equiv \delta \equiv 1(\bmod \sqrt{2}) .
\end{aligned}
$$


So $\alpha+\delta$ is congruent to zero modulo $\sqrt{2}$, and we can find an algebraic integer $w \neq 0$ such that $\alpha+\delta=w \sqrt{2}$. We also have $\alpha^{\prime}+\delta^{\prime}=-w^{\prime} \sqrt{2}$. Starting from (3.1) and using (1.1), we see that

$$
\begin{aligned}
& (\gamma r+\delta)^{2}+(\gamma s)^{2}=1=\left(\gamma^{\prime} r^{\prime}+\delta^{\prime}\right)^{2}+\left(\gamma^{\prime} s^{\prime}\right)^{2} \\
\Rightarrow & \left(\frac{\alpha+\delta}{2}\right)^{2}+(\gamma s)^{2}=1=\left(\frac{\alpha^{\prime}+\delta^{\prime}}{2}\right)^{2}+\left(\gamma^{\prime} s^{\prime}\right)^{2} \\
\Rightarrow & \frac{w^{2}}{2}+(\gamma s)^{2}=1=\frac{w^{\prime 2}}{2}+\left(\gamma^{\prime} s^{\prime}\right)^{2} .
\end{aligned}
$$

Let $x=\frac{w^{2}}{2}$ and $y=\frac{w^{\prime 2}}{2}$. Then we have

$$
0 \leq x, y \leq 1, \quad x y=\frac{\left(w w^{\prime}\right)^{2}}{4} \geq \frac{1}{4} .
$$

Considering the function

$$
h(x, y)=1-x-y+x y
$$

on the region defined in (3.2), we see that the critical point is at $(1,1)$ in the $x-y$ plane, a boundary point of the region. So the extreme values occur on the boundary of this region. On the boundary lines $x=1$ and $y=1$ the value of the function $h$ is zero. On the boundary curve $x y=1 / 4$ we have by the Arithmetic Mean Geometric Mean Inequality

$$
x+y \geq 2 \sqrt{x \cdot \frac{1}{4 x}} \geq 1,
$$

which implies

$$
h(x, y) \leq 1-1+1 / 4 \leq 1 / 4 .
$$

So $h$ is bounded between 0 and $1 / 4$ in the region (3.2). Hence

$$
\begin{aligned}
& (\gamma s)^{2}\left(\gamma^{\prime} s^{\prime}\right)^{2}=h(x, y) \leq 1 / 4 \\
\Rightarrow \quad & \left|\gamma \gamma^{\prime}\right| \leq \frac{1}{2} \frac{1}{s s^{\prime}} \leq \frac{1}{2} \frac{1}{.395} \leq 1.2658 .
\end{aligned}
$$

Thus $|N(\gamma)| \leq 1$, which is a contradiction.

Lemma 6. Let $\eta$ be an element of the modular group $\Gamma(O(\sqrt{2}))$ that fixes a point in $F(O(\sqrt{2}))$. Then

$$
|\gamma|,\left|\gamma^{\prime}\right|<3.85, \quad|\delta|,\left|\delta^{\prime}\right|<14.60
$$

If $|N(\gamma)|=1$, then additionally

$$
|\alpha|,\left|\alpha^{\prime}\right|<2.57, \quad|\beta|,\left|\beta^{\prime}\right|<12.90 .
$$

Proof. Similar to the proof for $\mathbf{Q}(\sqrt{5})$. When $|N(\gamma)|=1$ Gotzky has essentially demonstrated that the fixed point $\left(z, z^{\prime}\right)$ satisfies $\left|z z^{\prime}\right|=1$.

Running two computer scans, we obtain the following results. 
TABle II. Fixed Points in the Fundamental Domain for $\mathbf{Q}(\sqrt{2})$

\begin{tabular}{lccc}
\hline Point & Order & Matrix \\
\hline$(i, i)$ & 4 & $\left(\begin{array}{cc}0 & -1 \\
1 & 0\end{array}\right)$ & \\
$(i(\sqrt{2}-1), i(\sqrt{2}+1))$, & 4 & $\left(\begin{array}{cc}0 & \sqrt{2}-1 \\
-\sqrt{2}-1 & 0\end{array}\right)$ \\
$(-1 / 2+i \sqrt{3} / 2,-1 / 2+i \sqrt{3} / 2)$ & 6 & $\left(\begin{array}{cc}0 & -1 \\
1 & 1\end{array}\right)$ & \\
$\left(\frac{\sqrt{2}-1}{2}+i \sqrt{3} \frac{\sqrt{2}-1}{2},-\frac{\sqrt{2}+1}{2}+i \sqrt{3} \frac{\sqrt{2}+1}{2}\right)$ & 6 & $\left(\begin{array}{cc}0 & \sqrt{2}-1 \\
-\sqrt{2}-1 & 1\end{array}\right)$ \\
$(\sqrt{2} / 2+i \sqrt{2} / 2,-\sqrt{2} / 2+i \sqrt{2} / 2)$ & & $\left(\begin{array}{cc}\sqrt{2} & -1 \\
1 & 0\end{array}\right)$ & \\
$\left(\frac{-2+\sqrt{2}}{2}+i \frac{2-\sqrt{2}}{2},-\frac{2+\sqrt{2}}{2}+i \frac{2+\sqrt{2}}{2}\right)$ & 8 & $\left(\begin{array}{cc}\sqrt{2} & \sqrt{2}-1 \\
-\sqrt{2}-1 & 0\end{array}\right)$ \\
\hline
\end{tabular}

Theorem 6. There are 66 matrices in $\Gamma(O(\sqrt{2}))$ that correspond to possible fixed points in the fundamental domain $F$ for $\mathbf{Q}(\sqrt{2})$. The corresponding fixed points are at a distance of $\sqrt{2}$ and $\sqrt{6}$ from the origin. The product of the imaginary parts is either $1 / 2,3 / 4$ or 1 .

It is interesting to note that of the 66 matrices, twelve satisfy the condition that $|N(\gamma)|=2$, but each of these is a power of one of the other matrices for which $|N(\gamma)|=1$.

Working with a computer algebra system and further scanning yields the next result.

Theorem 7. There are a total of six $\Gamma(O(\sqrt{2}))$-inequivalent fixed points in the fundamental domain $F$ for $\mathbf{Q}(\sqrt{2})$. Two points have cyclic isotropy subgroups of order 4, two points have cyclic isotropy subgroups of order 6 and the final two points have cyclic isotropy subgroups of order 8.

Of the 66 matrices mentioned above, all are members of cyclic subgroups of order 4,6 or 8 . Clearly there must be a point in the fundamental domain fixed by a cyclic subgroup of the same order as any fixed point generated by the computer scan.

Table II lists a choice of inequivalent fixed points with corresponding matrices. Another program and a computer algebra system were used to show that the fixed points produced by these matrices were actually in the point set $F(O(\sqrt{2}))$.

Both tables agree with previous results on fixed points up to translation by a rational integer (see Gundlach [5, pp. 377, 378]). In that paper, there is a small typo in the last fixed point listed for $\mathbf{Q}(\sqrt{5})$ in the table for Satz 1, where $\sqrt{5}$ should be replaced by $\sqrt[4]{5}$. 
It should be remarked that Gundlach and Siegel use a different definition of the fundamental domain. While Gotzky was able to show $F(O(\sqrt{5}))$ is a fundamental domain, his proof breaks down for the case of $\mathbf{Q}(\sqrt{2})$. It is still the case, however, that $F(O(\sqrt{2}))$ contains a fundamental domain. Thus one may reasonably conjecture that $F(O(\sqrt{2}))$ is itself a fundamental domain for the modular group of $\mathbf{Q}(\sqrt{2})$. See Gotzky [3] pp. 420-421], Gundlach [5. pp. 371-375] and Siegel [10. pp. 186-208].

\section{The COMPutation}

The GNU C compiler and PUNIMAX, a variant of MAXIMA, were used on LINUX partitions on each of two PC's. The LINUX kernel version was 2.0.35 and the GNU C compiler version was 2.7.2.3. The most time-consuming aspect of the computation was searching for matrices that mapped one specific point into another specific point. This required using "for" loops nested 8 deep. Due to the wider range of permitted values in the $\mathbf{Q}(\sqrt{2})$ case, each search would take from 15 to 30 minutes. The lower time occured when using GNU C's level 2 optimization. The PC's had Pentium chips rated at $133 \mathrm{mhz}$ and $300 \mathrm{mhz}$, respectively. The RAM sizes were 32 megabytes and 64 megabytes, respectively.

Early versions of one of the scanning softwares were ported to Microsoft ${ }^{\circledR}$ and Borland ${ }^{\circledR} 16$ bit $\mathrm{C}$ compilers. Unfortunately the executables were extremely slow and three to four times larger than the GNU C executables. The Microsoft ${ }^{\circledR}$ debug version executable was so slow it was essentially impossible to use.

\section{ACKNOWLEDGMENTS}

The author would like to express his appreciation to the Mathematics Department of the University of Botswana for the use of their computing facilities, and to Paul Fisher for a suggestion. The author would like to thank B. Haible, the maintainer of PUNIMAX, for permitting its free use for academic purposes (Haible 6]). The author would also like to thank Harvey Cohn for many encouraging email communications.

\section{REFERENCES}

[1] H. Cohn, A Classical Invitation to Algebraic Numbers and Class Fields, Springer-Verlag, New York, 1978. MR 80c:12001

[2] J. I. Deutsch, Identities on Modular Forms in Several Variables Derivable from Hecke Transformations, Dissertation, Brown University, Providence, R.I., USA, 1986.

[3] F. Götzky, Über eine zahlentheoretische Anwendung von Modulfunktionen zweier Veränderlicher, Math. Ann. 100 (1928), 411-437.

[4] K. B. Gundlach, Die Bestimmung der Funktionen zu einigen Hilbertschen Modulgruppen, J. Reine Angew. Math. 220 (1965), 109-153. MR 33:1290

[5] K. B. Gundlach, Die Fixpunkte einiger Hilbertscher Modulgruppen, Math. Annalen 157 (1965), 369-390. MR 37:5153

[6] B. Haible, Private communication, 1997.

[7] F. Hirzebruch, Hilbert Modular Surfaces, Monographie No. 21 de L'Enseignement Mathématique, Geneva, Switzerland, 1973. MR 52:10750

[8] N. Koblitz, Introduction to Elliptic Curves and Modular Forms, Springer-Verlag, New York, USA,1984. MR 86c:11040 
[9] J-P. Serre, A Course in Arithmetic, Springer-Verlag, New York, USA, 1973. MR 49:8956

[10] C. L. Siegel, Advanced Analytic Number Theory, Tata Institute, Bombay, India, 1980. MR 83m:10001

Mathematics Department, University of Botswana, Private Bag 0022, Gaborone, BOTSWANA

E-mail address: deutschj_1729@yahoo.com 